\title{
CERATOCISTO ODONTOGÊNICO EM MAXILA: RELATO DE CASO CLÍNICO
}

ODONTOGENIC KERATOCYST IN MAXILLA: CLINICAL CASE REPORT

\section{Leticia Gonçalves Ferreira}

Aluna da Faculdade de Odontologia pela Universidade Federal Fluminense - UFF, Niterói/RJ, Brasil.

\section{Bruno Moura Mourão}

Cirurgião Buco-Maxilo-Facial do Serviço privado - Duque de Caxias/RJ, Brasil.

\section{Cícero Luiz Souza Braga}

Chefe do Serviço de Cirurgia e Traumatologia Buco-Maxilo-Facial do Hospital Federal de Ipanema, Rio de Janeiro/RJ, Brasil.

\section{Adriana Terezinha Novellino}

Professora da disciplina da Clínica Diagnóstica da Faculdade de Odontologia da Universidade Federal Fluminense, Niterói/RJ, Brasil.

\section{Marcelo José Pinheiro Guedes De Uzeda}

Professor da disciplina de Cirurgia Oral Menorda Faculdade de Odontologia da Universidade Federal Fluminense, Niterói/RJ, Brasil.

\section{Rodrigo Figueiredo De Brito Resende}

Professor da disciplina de Cirurgia Oral Menor da Faculdade de Odontologia da Universidade Federal Fluminense, Niterói/RJ, Brasil.

Instituição na qual o trabalho foi realizado:Hospital Federal de Ipanema, Rio de Janeiro/RJ, Brasil e Faculdade de Odontologia da Universidade Federal Fluminense, Niterói/RJ, Brasil.

Categoria:Relato de caso

Informações do autor principal:

Leticia Gonçalves Ferreira 
Rua Cruzeiro, 8, Pau Grande - Magé - RJ, Brasil. - CEP: 25933215

Email: leticiagsacento@gmail.com

\section{Resumo}

O Ceratocisto Odontogênico (CO)é uma lesão intraóssea benigna de origem odontogênica, que surge a partir dos restos celulares da lâmina dental; apresenta predominância de acometimento em mandíbula, principalmente com envolvimento do corpo posterior e ramo de mandíbula. Os COs podem ser encontrados em pacientes desde a infância até a velhice, todavia, mais da metade dos casos são diagnosticados em pessoas entre 10 a 40 anos de idade, sendo sua prevalência em homens. Tal lesão cística exibe ao exame radiográfico uma área radio lúcida, com margens escleróticas frequentemente bem definidas. Histologicamente manifesta revestimento epitelial composto por uma camada uniforme de epitélio escamoso estratificado, geralmente com 6 ou 8 camadas de espessura, podem ser observados ainda pequenos cistos, cordões ou ilhas satélites de epitélio odontogênico na cápsula fibrosa, a qual é tipicamente delgada e friável. O objetivo do presente trabalho é relatar o caso clínico do paciente BSM,sexo masculino, feoderma, 22 anos de idade, ASA I, que compareceu ao ambulatório de BucoMaxilo-Facial do Hospital Federal de Ipanema/RJapresentando uma lesão cística associada ao terceiro molar superior direito incluso. $O$ tratamento consistiu na enucleação e curetagem da lesãoem ambiente hospitalar sob anestesia geral, sem presença de intercorrências. A peça cirúrgica foi encaminhada ao Laboratório de Biotecnologia Aplicada da Universidade Federal Fluminense (LABA-UFF) para exame anatomohistopatológico, sendo confirmado seu diagnóstico inicial de $\mathrm{CO}$. O paciente recebeu alta no dia seguinte ao procedimento cirúrgico e segue em acompanhamento ambulatorial de 12 meses pela especialidade, sem presença clínica e imaginológica de recidiva da lesão.

Palavras-chave: Ceratocisto Odontogênico; Enucleação; Maxila

Abstract
The Odontogenic Keratocyst (CO) is a benign intraosseous lesion of odontogenic
origin, which increases from the cellular remains of the dental membrane; it presents
predominance of follow-up in the mandible, mainly with involvement of the posterior
body and mandible branch. OCs can be found in patients from childhood to old age,
however, more than half of the cases are diagnosed in people between 10 and 40 years
REVISTA FLUMINENSE DE ODONTOLOGIA-ANO XXVII- $\mathrm{N}^{\circ} 55$ - Janeiro / Julho 2021
ISSN 1413-2966/ D-2316 
of age, being its prevalence in men. Such a cystic lesion is detected in the radiographic examination of a radiolucent area, with sclerotic margins often quite reduced. Histologically, the epithelial lining composed of a uniform layer of stratified stratified epithelium, with 6 or 8 layers of thickness, can also be observed in small cysts, cords or satellite islands of odontogenic epithelium in the fibrous capsule, which is typically thin and friable. The objective of the present work is to relate the clinical case of BSM patient, male, feoderma, 22 years old, ASA I, who compared the cystic lesion associated with the included dental element to the buccomaxillofacial clinic of the Federal Hospital of Ipanema / RJ. Treatment consists of enucleation and curation of lesions in the hospital environment under general anesthesia, without the presence of complications. A surgical specimen was sent to the Laboratory of Applied Biotechnology by Universidade Federal Fluminense (LABA-UFF) for anatomo-histopathological examination, confirming its initial diagnosis of OC. The patient who was discharged the day after the medical procedure and is undergoing outpatient follow-up for 12 months, with no clinical and imaginative presence of injury recurrence.

Keywords: Odontogenic Keratocyst; Enucleation; Maxilla

\section{Introdução}

O Ceratocisto Odontogênico (CO), descrito primeiramente por Philipsen em 1956, é uma lesão intraóssea benigna de origem odontogênica que ocorre em aproximadamente $10 \%$ dos cistos mandibulares. São caracterizados por um comportamento agressivo com uma taxa de recorrência relativamente alta (BORGHESI A, ET AL. 2018). Há uma concordância geral de que o Ceratocisto Odontogênico surge a partir dos restos celulares da lâmina dental e apresenta um mecanismo de crescimento e comportamento biológico diferentes daqueles do Cisto Dentígero e do Cisto Radicular, que são os mais comuns(NEVILLE BW, ET AL. 2009).

A maioria dos autores acredita que o Cisto Dentígero e o Cisto Radicular continuam a crescer como conseqüência do aumento da pressão osmótica dentro do lúmen cístico. Tal mecanismo parece não ser verdadeiro para $\mathrm{CO}$, e seu crescimento pode estar relacionado a fatores desconhecidos, inerentes ao próprio epitélio ou à atividade enzimática na parede cística (NEVILLE BW, ET AL. 2009). A $4^{\mathrm{a}}$ edição da Classificação de Tumores de Cabeça e Pescoço publicada em 2017 pela World Health 
COPYRIGHT @ 2021 INTERNATIONAL JOURNAL OF SCIENCE DENTISTRY | AVAILABLE ONLINE http://www.periodicos.uff.br/index

Organization (WHO) tirou esta entidade patológica da categoria de neoplasia com a alegação de que, até o presente momento, não existem evidências suficientes para apoiar a origem neoplásica da lesão cística, sendo assim, reclassificado como Ceratocisto Odontogênico (WHO, 2017; SOLUK-TEKKESIN M, WRIGTH JM, 2017; BORGHESI A, ET AL. 2018).

Os Ceratocistos Odontogênicos podem ser encontrados em pacientes com idades variável, desde a infância até a velhice, mas cerca de $60 \%$ de todos os casos são diagnosticados em pessoas entre 10 a 40 anos. Há uma leve preferência para homens.A mandíbula é acometida em $60 \%$ a $80 \%$ dos casos, com uma marcante tendência para o envolvimento do corpo posterior e do ramo da mandíbula (NEVILLE BW, ET AL. 2009). Por outro lado, o sextante anterior, principalmente entre canino e incisivo lateral, e a região de terceiro molar são os locais de origem mais comunsna maxila(BORGHESI A, ET AL. 2018). Os COs exibem uma área radio lúcida, com margens escleróticas frequentemente bem definidas. São lesões grandes e podem se apresentar multiloculadas. Múltiplos Ceratocistos Odontogênicos podem estar presentes e tais pacientes devem ser avaliados em busca de outras manifestações da Síndrome do Carcinoma Nevoide Basocelular, ou Síndrome de Gorlin (NEVILLE BW, ET AL. 2009).

Esta lesão cística exibe tipicamente uma cápsula delgada, friável, que muitas vezes provoca dificuldades em ser enucleada do osso em um único pedaço. $\mathrm{O}$ revestimento epitelial é composto por uma camada uniforme de epitélio escamoso estratificado, geralmente com 6 ou 8 camadas de espessura. Pequenos cistos, cordões ou ilhas satélites de epitélio odontogênico podem ser observados na cápsula fibrosa. $O$ tratamento se dá através da enucleação e curetagem. Em contraste com outros cistos odontogênicos, os COs tendem, com frequência, recorrer após o tratamento, em vários estudos a taxa de recidiva varia de 5\% a 62\% (NEVILLE BW, ET AL. 2009).

O objetivo do presente trabalho é relatar um caso de Ceratocisto Odontogênico em maxila, cuja exérese da lesão foi executada e seguida de boa evolução pós- 
operatória. No follow-up de 12 mesesnão foiencontrado sinal clínico ou por imagem que sugerissem recidiva da lesão, sendo o paciente orientado sobre a necessidade de seu acompanhamento periódico pela especialidade.

\section{Considerações éticas}

Os procedimentos empregados neste trabalho seguiram os padrões éticos propostos pela Declaração de Helsinque de 1975, revisado em 2000. O paciente foi tratado no Hospital Federal de Ipanema através do ambulatório de Buco-Maxilo-Facial com os termos de consentimento livre e esclarecido e uso de imagem e dados para publicação científica assinados.

O estudo foi escrito seguindo as Diretrizes do Relatório de Caso Clínico (CARE, www.care-statement.org).

\section{Relato de Caso}

Paciente BSM, sexo masculino, feoderma, 22 anos de idade, ASA I, há mais de um ano na fila do Sistema de Regulação (SISREG) compareceu ao ambulatório de Bucomaxilofacial do Hospital Federal de Ipanema por encaminhamento do serviço particular solicitando avaliação de lesão cística associada ao terceiro molar superior direito incluso.

Ao exame clínico o paciente apresentou edema e dor local em hemi maxila direita, queixando-se de secreção amarelada e odor fétido em cavidade oral há aproximadamente 6 meses. Ao exame por imagem, radiografia panorâmica (Figura 1) e tomografia de face, foi evidenciada lesão radio lúcida, bem delimitada e associada ao terceiro molar superior direito incluso, tendo este sofrido deslocamento por conta da lesão. 


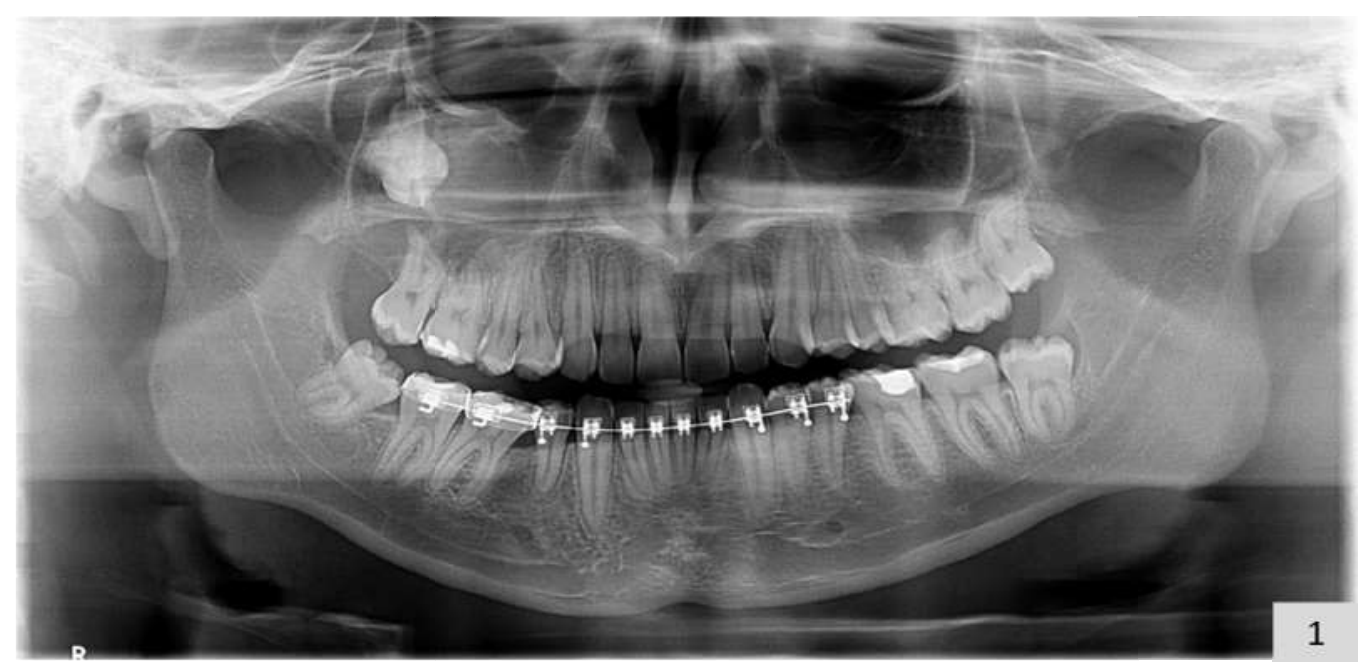

Figura 1- Radiografia panorâmica pré-operatória com lesão cística em hemi maxila direita.

Houve uma tentativa de realizar a biópsia incisional em ambulatório, todavia, mesmo sob sedação com benzodiazepínico paciente não foi colaborativo em decorrência de ansiedade exacerbada. Então, optou-se pela exérese da lesão no ambiente cirúrgico sob anestesia geral, procedimento também justificado por conta do tamanho da lesão, de aproximadamente 4 centímetros em seu maior diâmetro.

O tratamento foi realizado em decúbito dorsal sob anestesia geral, sendo a incisão mucoperiosteal em fundo de vestíbulo (Figura 2A e 2B) seguida de osteotomia a martelo e cinzel da parede lateral da maxila para acessar seio maxilar direitopermitindo, desta maneira,a abertura da loja cirúrgica (Figura2C), o que possibilitou melhor visualização para enucleação e curetagem da lesão cística e da mucosa de seio maxilar. 


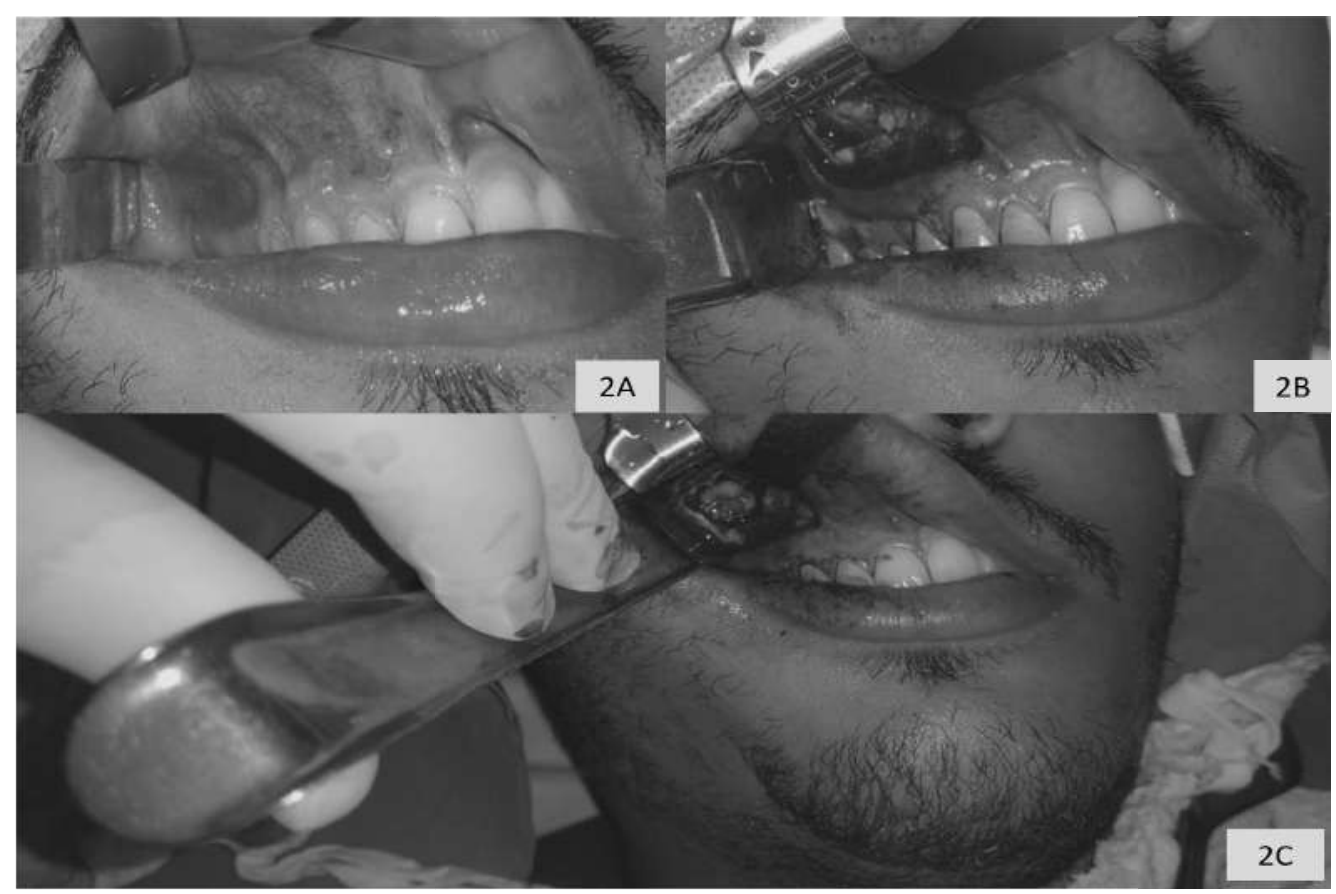

Figura 2- A. Afastamento de tecido referente ao primeiro quadrante; B. Incisão mucoperiosteal em fundo de vestíbulo; C. Loja cirúrgica com visão do elemento dentário 18.

O elemento dentário incluso $n^{\circ} 18$ foi removido e, então, feita a irrigação copiosa da cavidade cirúrgica com soro fisiológico estéril, foi realizada aplicação de hemostático (Figura 3B). Após síntese tecidual com Vicryl 3-0 (Johnson \& Johnson, Nova Jersey, EUA) (Figura 3C) paciente foi extubado, acordado e seguiu para observação. No pós operatório apresentou edema compatível com procedimento de ressecção de lesão, sem queixas álgicas. O paciente recebeu alta no dia seguinte ao procedimento cirúrgico.

A peça cirúrgica foi enviada ao Laboratório de Biotecnologia Aplicada da Universidade Federal Fluminense (LABA-UFF) para exame anatomohistopatológico, sendo notada presença de infiltrado inflamatório predominantemente crônico em grande parte da parede cística, e confirmado o diagnóstico de Ceratocisto Odontogênico.

O paciente retornoupara avaliação e remoção de sutura após 20 dias de procedimento cirúrgico,apresentando boa evolução. Para o follow-up de 7 meses foi solicitado pela REVISTA FLUMINENSE DE ODONTOLOGIA - ANO XXVII - N 55 - Janeiro / Julho 2021 
especialidade uma radiografia panorâmica (Figura 3D), na qual não foi observado sinais de recidiva da lesão, compatível com a avaliação clínica. Em seguida, foram dadas as orientações sobre a necessidade do retorno periódico para acompanhamento de possível recorrência da lesão cística. O mesmo segue em acompanhamento ambulatorial de 12 meses pela especialidade, sem presença clínica e imaginológica de recidiva da lesão.

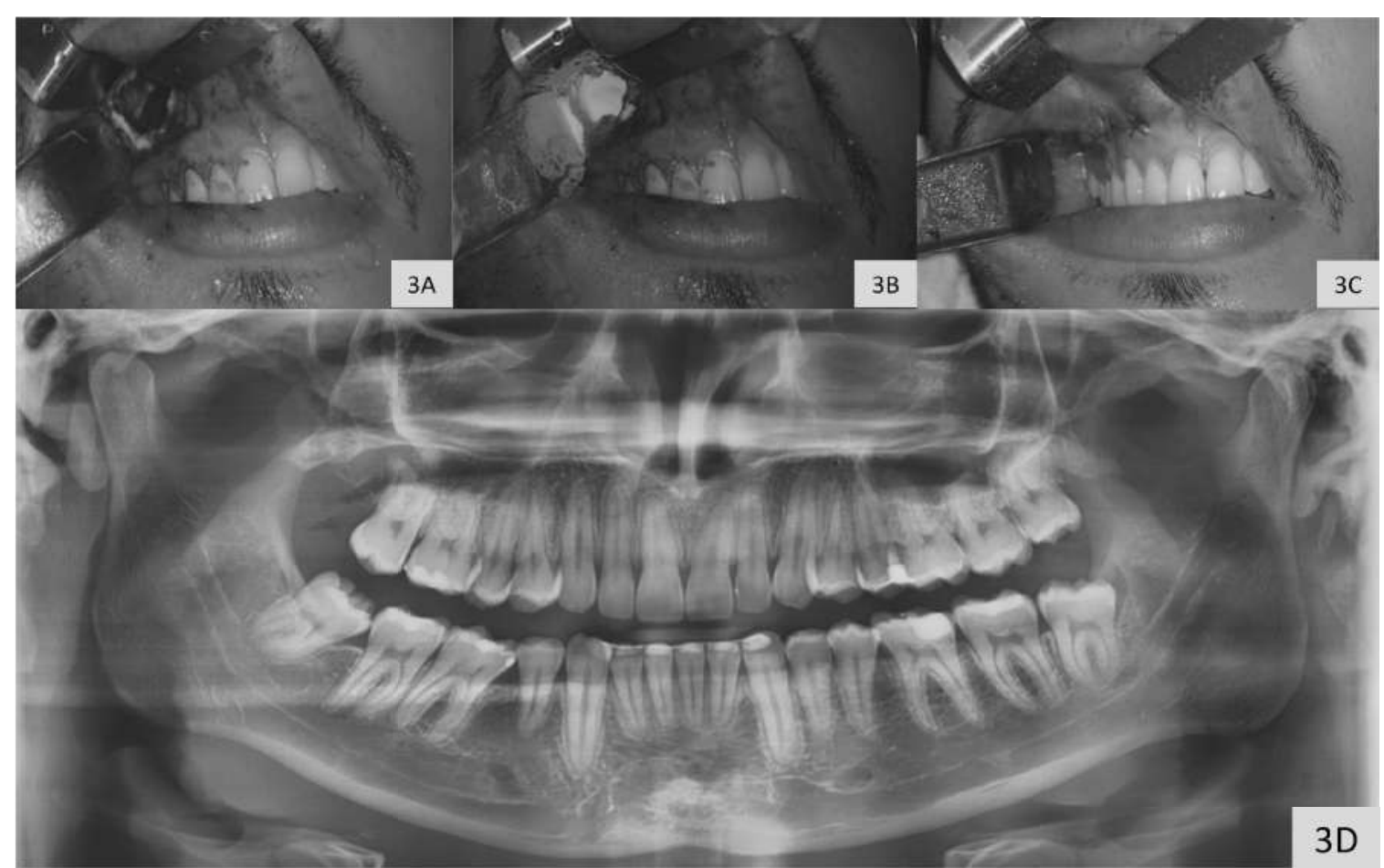

Figura 3- A. Após exérese da lesão cística e elemento dentário; B. Aplicação de hemostático; C. Síntese tecidual; D. Radiografia panorâmica de 7 meses de acompanhamento sem sinais de recidiva do Ceratocisto Odontogênico.

\section{Discussão}

O COé uma forma diferente de cisto odontogênico do desenvolvimento que merece consideração especial devido às suas características histopatológicas e comportamento clínico específicos. Geralmente quando de pequeno diâmetro são assintomáticos e descobertos somente durante o curso de um exame radiográfico. COs de grandes dimensões podem estar associados à dor, edema ou drenagem (NEVILLE BW, ET AL. 
2009; TAWFIK, ET AL.,2010; SIRIWARDENA, ET AL., 2012; CHRYSOMALI. ET AL., 2013; JOHNSON, ET AL., 2013; SEKERCI, ET AL., 2015).

Apesar de seu comportamento agressivo, na maioria dos casos, este tipo de cisto causa mínima expansão óssea por conta de sua propensão a se disseminar através do espaço intramedular,crescendo em uma direção ântero-posterior (NEVILLE BW, ET AL. 2009; BORGHESI A, ET AL. 2018). Diferente de outras lesões odonotogênicas que apresentam comportamento agressivo como ameloblastomas, COs pouco frequentemente causam reabsorção radicular de dente adjacente, e em $30 \%$ dos casos não apresentam relação com nenhuma estrutura dental adjacente (SEKERCI, ET AL., 2015; BORGHESI A, ET AL. 2018).

Os achados de imagem mais efetivos para fazer um diagnóstico provisório de $\mathrm{CO}$ são lesões osteolíticas uniloculares bem definidas em região posterior de maxila e grandes lesões osteolíticas mandibulares com poucos septos e mínima expansão bucolingual. Contudo, quando associado a dente impactado pode simular um cisto dentígero. Similarmente, quando multilocular e localizado no sextante posterior do ramo da mandíbula mimetiza o ameloblastoma. E pode ainda ser confundido com um cisto residual quando em posição periapical ou envolvendo uma área edentula. Como resultado, cisto dentígero, ameloblastoma e cisto radicular são considerados as lesões odontogênicas mais comuns no diagnóstico diferencial de CO (BORGHESI A, ET AL. 2018).

Apesar de poder haver suspeitada presença de um CO a partir dos exames clínicos e radiográficos, a confirmação histopatológica é requerida para o diagnóstico definitivo (NEVILLE BW, ET AL. 2009; SIRIWARDENA, ET AL., 2012; CHRYSOMALI. ET AL., 2013; JOHNSON, ET AL., 2013; TAWFIK, ET AL., 2010).

Várias opções cirúrgicas têm sido consideradas, incluindo apenas enucleação,ou esta combinada a medidas adjuvantes como osteotomia, solução de Carnoy, crioterapia, marsupialização, descompressão, ressecção marginal ou segmentar (BORGHESI A, ET AL. 2018). A maioria dos COs é tratada de forma similar a outros cistos odontogênicos, ou seja, através de enucleação e curetagem. Apesar de muitos CO recorrerem dentro do período de 5 anos após a cirurgia inicial, um número significativo de recidivas pode não 
COPYRIGHT @ 2021 INTERNATIONAL JOURNAL OF SCIENCE DENTISTRY | AVAILABLE ONLINE http://www.periodicos.uff.br/index

se manifestar até 10 ou mais anos após o procedimento cirúrgico original. Sendo o acompanhamento clínico e radiográfico em longo prazo, portanto, necessário (NEVILLE BW, ET AL. 2009).

No relato de caso supracitado foi realizada enucleação e curetagem da lesão cística com intuito sanar a infecção secundária causada pelo COe eliminar a entidade patológica, e, também, evitar sua recorrência. A intervenção cirúrgica mostrou-se eficiente no pósoperatório e no acompanhamento de 12 meses foi observada cicatrização do tecido sem sinais de recidiva da lesão.

\section{Conclusão}

A abordagem cirúrgica mostrou-se eficaz para eliminação da lesão, todaviasegundo a literatura,o acompanhamento periódico por um período maiordo paciente ainda é necessário.

\section{Referências Bibliográficas}

1. Borghesi A, Nardi C, Giannitto C, Tironi A, Maroldi R, Bartolomeo FD, Preda L. Odontogenic keratocyst: imaging features of a benign lesion with an aggressive behavior. Springer, Brescia, Insights into Imaging, v. 9, p. 883-897, 2018.

2. El-Naggar AK, Chan JKC, Grandis JR, Takata T, Slootweg PJ. WHO Classification of Head and Neck Tumours. 4a Ed. WHO, 2017.

3. Neville BW, Allen CM, Damm DD, Bouquot JE. Patologia Oral e Maxilofacial. 3a Ed. Rio de Janeiro: Elsevier; 2009.

REVISTA FLUMINENSE DE ODONTOLOGIA - ANO XXVII - N 55 - Janeiro / Julho 2021 
4. Philipsen HP. On keratocysts (cholesteatoma) in the jaw. Tandlaegebladet, 60, p.963-980, 1956.

5. Soluk-Tekkesin M, Wright JM. The World Health Organization Classification of Odontogenic Lesions: A Summary of the Changes of the 2017 (4th) Edition. TurkishJournalofPathology, Istambul, v.34, n.1, 2018.

6. Sekerci AE, Nazlim S, Etoz M, Deniz K, Yasa Y. Odontogenic tumors: a collaborative study of 218 cases diagnosed over 12 years and comprehensive review of the literature. Medicina Oral Patologia Oral y Cirugia Bucal, 20(1):e34-44, 2015.

7. Chrysomali E, Leventis M, Titsinides S, Kyriakopoulos V, Sklavounou A. Odontogenictumors. Journal of Craniofacial Surgery,24(5):1521-25, 2013.

8. Johnson NR, Savage NW, Kazoullis S, Batstone MD. A prospective epidemiological study for odontoge- nic and non-odontogenic lesions of the maxilla and mandible in Queensland. Oral Surgery, Oral Medicine, Oral Pathology and Oral Radiology, 115(4):515-22, 2013.

9. Siriwardena BS, Tennakoon TM, Tilakaratne WM. Relative frequency of odontogenic tumors in Sri Lanka: analysis of 1677 cases. Pathology, Research and Practice, 20(4):225-30, 2012.

10. Tawfik MA, Zyada MM. Odontogenic tumors in Dakahlia, Egypt: analysis of 82 cases. Oral Surgery, Oral Medicine, Oral Pathology and Oral Radiology, 109(2): e67-73, 2010 . 